\title{
Eficácia dos cones vaginais no fortalecimento do assoalho pélvico na incontinência urinária feminina pós- menopausa: estudo de casos
}

\author{
Effectiveness of vaginal cones in strengthening pelvic floor in \\ post-menopause female urinary incontinence: case report
}

\author{
Juliana Tornquist Holzschuh', Ana Cristina Sudbrack ${ }^{2}$ \\ 'Autora para correspondência. Universidade de Santa Cruz do Sul. Santa Cruz do Sul, Rio de Grande Sul, Brasil. \\ ORCID: 0000-0002-0299-2926. julianaholzschuh@gmail.com \\ 2Universidade de Santa Cruz do Sul. Santa Cruz do Sul, Rio de Grande Sul, Brasil. ORCID: 0000-0001-7426-7443. acs@unisc.br
}

\begin{abstract}
RESUMO | INTRODUÇÃO: A incontinência urinária (IU), pode afetar ambos os sexos, porém apresenta alta prevalência no sexo feminino, sendo as mulheres, na terceira idade, as mais acometidas. A IU afeta a qualidade de vida (QV), pois causa desconforto, redução da autoconfiança, alteração do comportamento, interferência na sexualidade, no convívio social, na saúde física e emocional. OBJETIVO: Avaliar o uso de cones vaginais no fortalecimento do assolho pélvico (AP) em mulheres com incontinência urinaria pós-menopausa. MÉTODOS: Estudo quantitativo, de delineamento observacional exploratório do tipo estudo de casos, realizado com 2 mulheres inscritas para tratamento de IU. Foi realizado a aplicação do questionário International Consultation on Incontinense Questionnaire (ICIQ-SF) e avaliação funcional do AP por meio do perineômetro, antes e após 10 sessões, realizadas 3 vezes por semana, durante 45 minutos, através de um programa de tratamento com uso de cones vaginais e cinesioterapia, associando aos exercícios de Kegel. RESULTADOS: Quanto ao ICIQ-SF, a paciente 1, na avaliação pontou o escore de 10 pontos (muito grave) e na reavaliação 2 (leve impacto). A paciente 2 , inicialmente pontuou 9 (grave impacto) e após o tratamento, 1 (leve impacto).. Na avaliação da contração muscular a paciente 1 obteve 40 sauers (normal) e passou para 44 (bom). A paciente 2 passou de 16 sauers (regular) para 28 (normal). CONCLUSÃO: Os cones vaginais beneficiaram mulheres com incontinência urinário de esforço, fortalecendo os músculos do assoalho pélvico, proporcionando meIhora na qualidade de vida.
\end{abstract}

PALAVRAS-CHAVE: Incontinência urinária. Menopausa. Fortalecimento. Fisioterapia. Assoalho pélvico.
ABSTRACT | INTRODUCTION: Urinary incontinence (UI) can affect both sexes, but it has a high prevalence in females, and women in the third age are the most affected. UI affects quality of life (QOL) because it causes discomfort, reduced self-confidence, behavioral change, interference with sexuality, social life, physical and emotional health. OBJECTIVE: To evaluate the use of vaginal cones in strengthening pelvic stinging (AP) in women with postmenopausal urinary incontinence. METHODS: A quantitative, observational exploratory case study design, conducted with 2 women enrolled for UI treatment. The International Consultation on Incontinence Questionnaire (ICIQ-SF) was applied and the functional assessment of the AP by the perineometer, before and after 10 sessions, performed 3 times a week, for 45 minutes, through a treatment program. of vaginal cones and kinesiotherapy, associated with Kegel exercises. RESULTS: Regarding the ICIQ-SF, patient 1 , in the evaluation, scored 10 points (very severe) and reevaluation 2 (mild impact). Patient 2 initially scored 9 (severe impact), and after treatment 1 (mild impact) both had stress $\mathrm{UI}$. In the evaluation of muscle contraction, patient 1 obtained 40 sauers (normal) and went to 44 (good). Patient 2 went from 16 sauers (regular) to 28 (normal). CONCLUSION: The vaginal cones benefited women with stress urinary incontinence, strengthening the pelvic floor muscles, providing improved quality of life.

KEYWORDS: Urinary incontinence. Menopause. Fortification. Physiotherapy. Pelvic floor. 


\section{Introdução}

A Sociedade Internacional de Continência caracteriza a incontinência como toda perda involuntária de urina, ocorrendo por uma disfunção do trato urinário inferior que advém por alteração fisiológica da micção ou nas estruturas de sustentação dos órgãos que participam da micção. Essa condição afeta a qualidade de vida das mulheres, pois causa uma série de consequências como desconforto, redução na autoconfiança, alteração do comportamento, interferência na sexualidade, no convívio social, na saúde física e emocional3,16,6,25.

Essa disfunção uroginecológica pode ser classificada em três tipos, considerando os sintomas: incontinência urinária de esforço (IUE), que é a perda urinária relacionada com atividades e manifestações que aumentam a pressão intra-abdominal; incontinência urinária de urgência (IUU), definida como perda involuntária de urina associada com um forte desejo de urgência para urinar, e incontinência mista (IUM), quando existe associação dos sintomas de incontinência de esforço e de urgência ${ }^{17,8}$.

São muitos os fatores de risco que podem levar ao desenvolvimento da IU, tais como idade avançada, parto vaginal, redução dos níveis de estrogênio na menopausa, prolapso vesical ou uterino, constipação intestinal, raça branca, obesidade, cirurgias ginecológicas ${ }^{9,6,25}$.

Na menopausa as causas da IU são decorrentes da mudança na função ovariana, com redução dos níveis de estrogênio, levando a diminuição do trofismo e da vascularização dos músculos que compõem o assoalho pélvico (MAP). O MAP se torna mais delicado, seco e menos elástico, tornando-se passível de estresses que possam levar a IU25.

O tratamento fisioterapêutico é indicado como primeira opção pela Sociedade Internacional de Continência ${ }^{2}$. A fisioterapia dispõe de diversos tipos de tratamentos para IU, que buscam aumentar o fortalecimento do assoalho pélvico; entre eles se destaca os cones vaginais. O cone vaginal, foi proposto por Plevnik para o fortalecimento resistido e progressivo dos músculos do assoalho da pelve. Essa terapia proporciona uma percepção da região perineal, aumentando a força muscular do assoalho pélvico e o recrutamento de fibras musculares do tipo I e tipo II $^{11}$.

Perante a contextualização exposta, o objetivo do estudo foi avaliar o uso de cones vaginais no fortalecimento do assolho pélvico em mulheres com incontinência urinaria pós - menopausa.

\section{Métodos}

\section{Desenho do estudo}

Trata-se de um estudo quantitativo, de delineamento observacional exploratório do tipo estudo de casos $^{7}$. Os dados foram coletados no período de abril e maio de 2019. Esta pesquisa foi devidamente aprovada pelo Comitê de Ética em Pesquisa CAAE 94112518.1.0000.5343. E as participantes após o aceite, assinaram o Termo de Consentimento Livre e Esclarecido.

\section{Participantes}

A mostra não probabilística da pesquisa foi constituída por conveniência, composta por mulheres no período pós-menopausa, inscritas para tratamento de IU, no primeiro semestre do ano de 2019, com disponibilidade de comparecer às coletas de dados e que não haviam realizado tratamento prévio. Foram critérios de exclusão mulheres no pós-parto com incontinência urinária, mulheres que apresentavam perda fecal e aquelas que já haviam realizado cirurgia reconstrutora de assoalho pélvico.

\section{Procedimentos metodológicos}

Inicialmente foi realizada uma avaliação funcional do assoalho pélvico, através do aparelho perineômetro digital da marca Kroman. O perineômetro de Kegel é um dispositivo sensível à pressão, que é inserido na vagina e prevê valores numéricos para a contração muscular ${ }^{11}$. A Escala Sauer possui uma equivalência 
em libras de pressão, onde, 28 Sauers equivalem a uma musculatura saudável sendo classificado como, ruim de 0-10 Sauers, regular de 11-25, normal de 2640, bom de 41-60, excelente de 61-80 e extraordinário de $81-100^{23}$.

Foi solicitado que a participante se posicionasse sobre a maca em decúbito dorsal com os joelhos flexionados, o perineômetro foi inserido na vagina com utilização de preservativo. Solicitado que a participante realizasse a contração da musculatura, onde o valor apontado no equipamento foi considerado.

Ao final da avaliação foi realizado o preenchimento do questionário International Consultation on Incontinense Questionnaire (ICIQ-SF) para avaliar o impacto da IU na QV e qualificar a perda urinária das participantes. Este instrumento foi traduzido para o português, adaptado e validado por Tananini et al. (2004). Composto por quatro questões que avaliam a frequência, a gravidade e o impacto da IU na Qualidade de Vida (QV) e um conjunto de oito itens de autodiagnostico, relacionados às causas ou a episódios de IU vivenciadas pelas participantes ${ }^{14}$.

Os escores do ICIQ-SF são obtidos por valores numéricos, em que a pontuação total varia de 0 a 21 pontos, sendo que, quanto maior a soma de pontos, maior a gravidade e o impacto da IU na QV. O impacto sobre a QV é classificado sendo: zero (0) ponto, nenhum impacto; de um a três pontos, leve impacto; de 4 a 6 pontos, moderado; de 7 a 9 pontos, grave; e, de 10 ou mais pontos, muito grave ${ }^{14}$.

Na sequência foi iniciado o programa de tratamento com uso de cones vaginais e cinesioterapia, associando aos exercícios de Kegel. Os cones são dispositivos de formas e tamanhos iguais e peso que variam de $25 \mathrm{~g}$ a $75 \mathrm{~g}$, e que são inseridos na vagina ${ }^{11}$. O programa de exercícios foi realizado três vezes na semana com duração de 45 minutos, totalizando 10 sessões, onde a participante foi orientada de como utilizar o cone vaginal, assim como a escolha do cone adequado, aquele em que a participante conseguiu segurar dentro da vagina por 1 minuto na posição ortostática, conforme adquiriu força o peso foi substituído.
O programa de exercícios foi realizado com cada participante, onde incluía subir e descer degraus, realizar agachamento com apoio, caminhada lateral com faixa elástica, deambular associando exercícios de contração em diferentes posturas, exercícios de Kegel para fortalecimento da musculatura pélvica, com contração de 5 segundos e relaxamento por 10 segundos. Realizados com o cone vaginal inserido na vagina.

Ao final do tratamento proposto, as pacientes foram submetidas à reavaliação funcional do assolho pélvico com perioneômetro e aplicado o questionário ICIQ-SF para comparação dos dados antes e após a intervenção.

\section{Análise dos dados}

Os dados da pesquisa foram tabulados e analisados através do Microsoft Office Excel em ilustrações (tabelas ou gráficos) para decorrente discussão dos resultados fundamentados no referencial teórico.

\section{Resultados}

Participaram do estudo duas mulheres em período pós-menopausa, sendo a Paciente 1 (66 anos) e a Paciente 2 (71 anos). Em relação ao tipo de IU encontrado em nosso estudo, ambas apresentavam a de esforço.

Na Tabela 1 estão apresentados os dados das avaliações pré e pós 10 sessões do programa de exercício, em que ambas as pacientes apresentaram aumento da força de contração muscular. 


\begin{tabular}{lcc}
\hline PERINEÔMETRO & Avaliação & Pós tratamento \\
\hline PACIENTE 1 & 40 & 44 \\
$\quad$ Contração muscular (sauers) & Normal & Bom \\
$\quad$ Classificação & & 28 \\
\hline PACIENTE 2 & 16 & Normal \\
$\quad$ Contração muscular (sauers) & Regular & \\
Classificação & & \\
\hline
\end{tabular}

Fonte: Dados da pesquisa, 2019.

Na Tabela 2, os resultados do ICIQ-SF foram positivos, havendo melhora da qualidade de vida, bem como redução da perda de urina tanto na Paciente 1 como na Paciente 2.

Tabela 2. Avaliação da qualidade de vida pré e pós 10 sessões

\begin{tabular}{lcc}
\hline ICIQ-SF & Avaliação & Pós tratamento \\
\hline PACIENTE 1 & & 2 \\
Escore (pontos) & 10 & Leve impacto \\
Classificação & Muito grave & Nunca \\
Quando você perde urina? & Tusso ou espirro & 1 \\
PACIENTE 2 & & Leve impacto \\
Escore (pontos) & 9 & Nunca \\
Classificação & Grave impacto & \\
Quando você perde urina? & Em atividades físicas & \\
\hline
\end{tabular}

ICIQ-SF: International Consultation on Incontinense Questionnaire.

Fonte: Dados da pesquisa, 2019.

Através da Figura 1 podemos verificar que apesar do bom desempenho das duas pacientes encontrado pelas avaliações, a Paciente 1 evoluiu para um cone de maior peso primeiro que a Paciente 2.

Figura 1. Evolução dos cones vaginais de acordo com as sessões

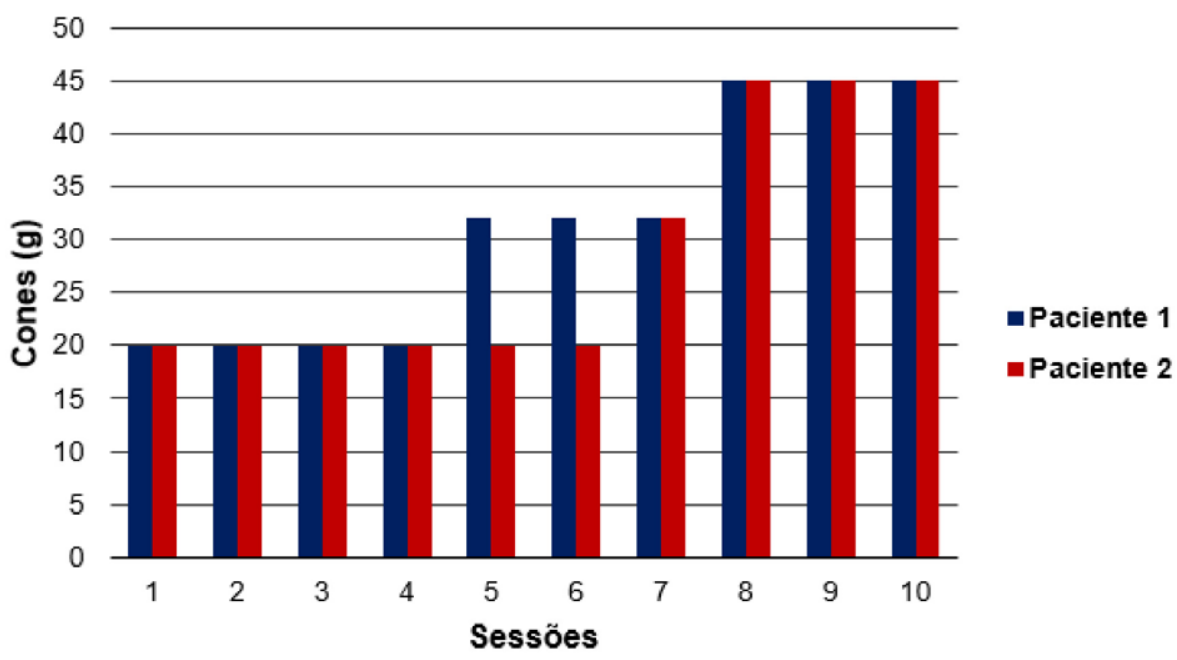

g: gramas.

Fonte: Dados da pesquisa, 2019 


\section{Discussão}

Esta estudo de casos teve o objetivo de avaliar o uso de cones vaginais no fortalecimento do assolho pélvico em duas mulheres com incontinência urinaria pós-menopausa. Em relação ao tipo de IU encontrado em nosso estudo, ambas apresentavam a de esforço, este achado está de acordo com a literatura, uma vez que estudos apontam que a IUE é o tipo mais frequente de IU no sexo feminino ${ }^{10,22}$.

São várias as técnicas que vêm sendo utilizadas, apesar de não haver grande consenso na literatura. Dentre elas, encontram-se os exercícios específicos para o AP (diversas dosagens), sendo esta auxiliada ou não por eletroestimulação e cones vaginais. Uma revisão narrativa da literatura apontou que a fisioterapia pélvica, principalmente no tratamento da IU, apresenta resultados promissores, tanto na meIhora da QV quanto na redução das perdas urinárias ${ }^{4}$. Um outro estudo empírico também verificou redução da frequência de perda de urina e ausência de desconforto ao realizar suas atividades diárias, e ainda foi observado melhora da força muscular do AP com aumento do grau de contração².

Além do uso em IU, os exercícios de fortalecimento do AP utilizando cones vaginais têm apresentado bons resultados na recuperação da musculatura após o parto ${ }^{12}$.

Nossos resultados referentes à QV são semelhantes aos evidenciados por Castro et al..$^{2}$, demonstrando eficácia na melhora dos escores de QV das pacientes. Padilha et al. ${ }^{14}$, apesar de não realizar intervenção, ao utilizar o ICIQ-SF para avaliar QV de 44 mulheres com IU, observaram que $a \mathrm{QV}$ em relação às perdas urinárias se mostrou de fraca a moderada. Por este fato, podemos verificar que nos casos relatados no presente estudo, se superou os escores esperados para a idade e condição clínica.

Souza et al. ${ }^{20}$ ao estimar a prevalência de IU em 233 mulheres no climatério e investigar os fatores associados, encontraram alta frequência de relatos de perda em pequena quantidade de urina (75\%), sendo sugerido a melhor capacitação de serviços de atenção primária para que haja uma maior qualidade tanto na prevenção como no tratamento da IU.
Silva et al. ${ }^{19}$ realizaram um estudo de caso em que associaram exercícios de Kegel aos cones vaginais, sendo realizadas 10 sessões de fisioterapia três vezes por semana, com duração de uma hora. Desta forma, semeIhante ao nosso estudo, a paciente inicialmente usou o cone rosa de $20 \mathrm{~g}$, passando pelo amarelo de $32 \mathrm{~g} \mathrm{e}$ evoluindo até o cone branco de $45 \mathrm{~g}$, obtendo como resultados a melhora do grau de força muscular ${ }^{19}$.

Entretanto. o estudo de Santos et al. ${ }^{18,}$ ao realizarem um estudo comparando duas técnicas em 45 mulheres com IUE, que consistiram em cones vaginais e eletroestimulação funcional do $\mathrm{AP}$, durante 4 meses. Obtiveram melhora significativa na QV e na diminuição da perda urinária, não havendo diferença significativa entre as técnicas no tratamento de IUE ${ }^{18}$. Portanto, parece que importa se o tratamento será por exercícios ou eletroestimulação, podendo ser escolhida qualquer uma das modalidades de acordo com a experiência do profissional, recursos disponíveis e preferencia das pacientes.

Apesar de um estudo ter envolvido 39 pacientes com síndrome da bexiga hiperativa que corresponde a outra condição clínica, Yüce et al. ${ }^{24}$ ao compararem a eficácia dos exercícios musculares do AP associados ao uso de cones vaginais também encontraram melhora dos sintomas e da QV após intervenção. A proposta de utilizar o cone vaginal para o fortalecimento resistido e progressivo dos músculos do assoalho, tem sido efetiva. Essa terapia proporciona uma percepção da região promovendo um feedback sensorial, levando os músculos do assoalho pélvico a se contraírem com mais vigor e melhor percepção $0^{11,15}$.

Para a escolha do cone adequado, as pacientes iniciam o treinamento com o cone que conseguiram manter dentro da vagina por um minuto na posição ortostática, após o treinamento os pesos se tornam crescentes. A contração involuntária ocorre durante o uso dos cones, ocorrendo momentos de contração e relaxamento, impedindo a fadiga muscular e a saída do cone da vagina. Assim os cones atuam na estimulação e no recrutamento das fibras do tipo I (contração lenta) e do tipo || (contração rápida), melhorando a propriocepção da musculatura pélvica e estimulando aumento de força muscular ${ }^{11,15}$. 
Para aumentar o conhecimento de formas para evitar e tratar a ocorrência de IU, Oblasser et al. ${ }^{12}$ realizaram uma revisão sistemática sobre o assunto e encontraram evidências bastante limitadas. Este fato justifica a produção de estudo de casos que podem adicionar informações às evidências atuais.

Ressalta-se que é de extrema importância a busca precoce por tratamento adequado da $\mathrm{IU}^{13}$. Desta forma, Del Pino et al. ${ }^{5}$ reforçam que a taxa de adesão ao treinamento dos músculos do AP em pacientes com IU feminina são reduzidas, apresentando a necessidade de estratégias de melhor acesso e adesão da terapêutica.

Como fatores limitantes desde trabalho, podemos citar o número reduzido de participantes, não permitindo a generalização dos resultados obtidos, se fazendo necessário a continuidade do trabalho buscando uma amostra maior capaz de potencializar a efetividade do uso dos cones no aumento da força muscular do AP.

\section{Conclusão}

Diante do exposto, foi possível verificar que a utilização dos cones vaginais auxiliaram e beneficiaram mulheres no período pós-menopausa com IUE, fortalecendo os músculos do AP, e proporcionando uma melhora na QV, evidenciada pelos resultados do questionário ICIQ-SF.

\section{Contribuições das autoras}

Holzschuh JT e Sudbrack AC participaram da concepção, delineamento, da coleta de dados da pesquisa, análise estatística dos dados da pesquisa, interpretação dos resultados e redação do artigo científico.

\section{Conflitos de interesses}

Nenhum conflito financeiro, legal ou político envolvendo terceiros (governo, empresas e fundações privadas, etc.) foi declarado para nenhum aspecto do trabalho submetido (incluindo, mas não se limitando a subvenções e financiamentos, participação em conselho consultivo, desenho de estudo, preparação de manuscrito, análise estatística, etc.).

\section{Referências}

1. Caetano AS, Tavares MCGCF, Lopes MHBM. Proposta de atividades físicas para mulheres com incontinência urinária de esforço. Lecturas: Educación Física y Deportes. 2004;10(76):1-10.

2. Castro LA, Machado GC, Trindade APNT. Fisioterapia em mulheres com incontinência urinária-relatos de caso. Revista UNINGÁ. 2019;56(S4):39-51.

3. Almeida ALR, Marsal AS. A influência da fisioterapia aplicada no tratamento da incontinência urinária de esforço em mulheres: estudo da eficácia da cinesioterapia. Visão Universitária.2015;3(1):109-128.

4. Oliveira AHFV, Vasconcelos LQP, Nunes EFC, Latorre GFS. Contribuições da fisioterapia na incontinência urinária no climatério. Ver Ciênc Méd. 2017;26(3):127-133. doi: 10.24220/2318-0897v26n3a3842

5. Pino DV, Martinéz RC. La adherencia al entrenamiento muscular del suelo pélvico en las mujeres con incontinencia urinaria. Revisión sistemática de la literatura. Fisioterapia, 2019. doi: 10.1016/j.ft.2019.03.005

6. Dias EM, Dias JM, Santos LM, Bitencourt ACP, Oliveira NKS, Nunes GGF. Cinesioterapia como tratamento da incontinência urinária de esforço-estudo de caso. Revista Eletrônica Estácio Saúde. 2016;5(1):61-72.

7. Goldim JR. Manual de Iniciação à Pesquisa em Saúde. 2 ed. revisada e ampliada. Porto Alegre: DaCasa; 2000.

8. Inhoti PA, Silva ES, Oliveira DV, Bertolini SMMG. Cinesioterapia uroginecológica na incontinência urinária de mulheres idosas fisicamente ativas. Revista Inspirar Movimento \& Saude. 2018;16(2):38-47.

9. Marques LP, Schneider IJC, Giehl MWC, Antes DL, D `orsi E. Fatores demográficos, condições de saúde e hábitos de vida associados à incontinência urinária em idosos de Florianópolis, Santa Catarina. Revista Brasileira de Epidemiologia. 2015;18(3):595-606. doi: 10.1590/1980-5497201500030006

10. Minassian VA, Devore E, Hagan K, Grodstein F. Severity of urinary incontinence and effect on quality of life in women, by incontinence type. Obstetrics and gynecology. 2013;121(5):10832013. doi: 10.1097/AOG.0b013e31828ca761

11. Moreno AL. Fisioterapia em uroginecologia. 2 ed. Barueri: Manole; 2009.

12. Oblasser C, Christie J, McCourt C. Vaginal cones or balls to improve pelvic floor muscle performance and urinary continence in women post partum: A quantitative systematic review. Midwifery. 2015;31(11):1017-25. doi: 10.1016/j.midw.2015.08.011 
13. Oliveira BS, Corbage RN. Fisioterapia no tratamento da incontinência urinária feminina: revisão bibliográfica. 2017. 34f. [Monografia]. São Paulo:Faculdade de Pindamonhangaba; 2017.

14. Padilha JF, Silva AC, Mazo GZ, MARQUES CMG. Investigação da qualidade de vida de mulheres com incontinência urinária. Arquivos de Ciências da Saúde da UNIPAR. 2018;22(1):43-48. doi: 10.25110/arqsaude.v22i1.2018.6302

15. Palma, PCR. Urofisioterapia: aplicações clínicas das técnicas fisioterapêuticas nas disfunções miccionais e do assoalho pélvico. 2 ed. São Paulo: Reproset, 2014.

16. Prado NF, Jucatelii VVB, Valente PHF, Alves FAVB, Mendonça RMC, Costa AP, Nogueira MS. Contribuição da assistência fisioterapêutica na correção da incontinência urinária: uma revisão bibliográfica. Revista Eletrônica Faculdade Montes Belos. 2016:8(3):113-179.

17. Santos ACCC, Dias SFL, Barbosa APB, Silva CML, Rocha VLS. Atuação do método Pilates no fortalecimento do assoalho pélvico na incontinência urinaria de esforço. ReonFacema. 2017;3(3):617623.

18. Santos PFD, Oliveira E, Zanetii MRD, Arruda RM, Sartori MGF, Girão MJBC, Castro RA. Eletroestimulação funcional do assoalho pélvico versus terapia com os cones vaginais para o tratamento de incontinência urinária de esforço. Rev Bras Ginecol Obstet. 2009;31(9):447-52. doi: 10.1590/50100-72032009000900005

19. Silva AMN, Oliva LMP. Exercícios de Kegel associados ao uso de cones vaginais no tratamento da incontinência urinária: estudo de caso. Scientia Medica. 2011;21(4):173-176.

20. Souza JO, Mascarenhas CS, Nascimento ACP, Gonçalves AS, Pereira LC, Kuraiem MAP, Cruz AT. A prevalência de incontinência urinária em mulheres na pós-menopausa. Revista Univap. 2015;21(37):31-42. doi: 10.18066/revistaunivap.v21i37.288

21. Tamanini JTN, Dambros M, D `ancona CAL, Palma PCR, Netto JR NR. Validação para o português do" Internacional Consultation on Incontinence Questionnaire-Short Form"(ICIQSF). Rev Saúde Pública 2004;38(3):438-44. doi: 10.1590/S003489102004000300015

22. Wiśniewska B, Marciniak A, Rutkowska-Nawrocha J, Ciecwiez M, Szydlowska I, Starczewski A. Urinary stress incontinenceone of basic diseases of modern society. Pol Merkur Lekarski.2015;38(223):51-54.

23. Wollmann L, Goulart CL, Mansour KMK, Cerentini TM, Carvalho LL, Sudbrack AC. Influência da prática de futsal amador na força muscular do assoalho pélvico. Rev Pesq Fisio. 2018;8(3):313-318. doi: 10.17267/2238-2704rpf.v8i3.1981
24. Yüce T, Dökmeci F, Çetinkaya ŞE. A prospective randomized trial comparing the use of tolterodine or weighted vaginal cones in women with overactive bladder syndrome. European Journal of Obstetrics \& Gynecology and Reproductive Biology.2016;197:91-7. doi: 10.1016/j.ejogrb.2015.11.034

25. Zezi B, Camargo HS, Souza JC. Prevalência e impacto da incontinência urinária na qualidade de vida em mulheres no período pós-menopausa. Revista FisiSenectus2016;4(2):12-21. doi: 10.22298/rfs.2016.v4.n2.3484 